\title{
General index
}

In preparing the indices, largely from lemmata supplied by Victor Skretkowicz, I have been greatly assisted by Dr Stefanie Lethbridge of Freiburg University and Ms Pia Prestin of Tübingen University. JBL.

Accession Day 35, 39-42, 45-6, 53, $63,123,170,193,216,255$

Acciaiuoli, Donato 226

Achilles 69, 112, 130, 160, 163, 193, 311

Achilles Statius 70, see also Achilles Tatius

Achilles Tatius 1, 2, 4-5, 10, 12, $15-16,19,28,34-6,55,63$, 64-110, 113-14, 116-17, 134, $168,181,184,198,203,206$, $210,219,254,260,284,289$, 295, 315, 334

Leukippe and Kleitophon 10, 15, 55, 63-5, 71-4, 80, 95, 103, 110-11, 116, 132, 183-5, 199, 204, 209, 217, 222, 263, 306

Adlington, William 24

Aeneas 186, 301

Agamemnon 33, 130

Agesilaus 230

Agricola, Rodolphus 13, 16

Agrippa, Henry Cornelius 120

Ainianes 130

Alba, Fernando Álvarez de Toledo y Pimentel, third Duke of 68, 177

Alcyone 184

allegory (allegorical, allegorically, allegorise, allegorised) see rhetoric
Allègre, Antoine 227

All's Well that Ends Well see Shakespeare

Amadis de Gaule (Amadis) 31, 122, 127, 182, 183, 196, 199, 203, 209, 219, 224, 277, 299, 334, 336

Amazon (Amazonian) 169, 180, 196

Amyot, Jacques 2, 4-6, 19-20, 22, 24, 28-34, 36, 38-9, 41, 43-4, 46, 48-63, 65, 69-71, 74, 88, 91, $110,114,117-19,127-9,132$, 134-44, 147-8, 151-62, 164-5, 169, 206-7, 225-31, 234, 240, $245,254,257,259,270,336-7$ see also Saint-Corneille de Compiègne, Abbot of

Andromeda 22, 111-12, 117, 130, 160, 163, 195

An Ethiopian Story see Heliodorus Anglican 2, 269, 338

Anjou, Duc d' see Valois, François Hercule, Duc d'Anjou

Anna (Anne) of Denmark 268 anti-romance 185, 246, 247, 249, 298, 300

Antony and Cleopatra see Shakespeare Antony, Marc 234, 245

Antwerpes Vnitye 176 
Aphrodite 75-6, 97, 99, 105, 109, 199, 202, 223

Aphthonius 4, 12-14, 16, 22, 211

Apollo 205, 258, 262, 293, 304

aposiopesis see rhetoric

Appian 206, 234-6

Apuleius 22-3

Arbaleste, Charlotte, Madame Du Plessis-Mornay see Du PlessisMornay, Madame Charlotte Arbaleste

Arcadia see Sidney, Sir Philip

Arcadius 171

Argus 201

Ariosto 154

Aristotle 10, 16-17

Artemis 101-2, 107-8, 199

Arthur of Little Britaine 122

Astarte 75-6

Astraea 173

Astrophil and Stella see Sidney, Sir Philip

As You Like It see Shakespeare

Athene 131, 195

Attic (Atticist) see Rhetoric

Attowell, George 210

Aubrey, John 306

Augustus 237, 249-50, 266

see also Octavius

Aurora 282

Austria, Elisabeth of, Queen of France 30

B. [Barker?], W. 235

Bacchus 41

Bandello, Matteo 19, 69

Banqueting House 43

Barclay, John 305-6

Bardus 172

Baron, Robert 312

Barreiros (Varreiro, Vacerius), Gaspar 172

Basel, Confession of 114

Baynard's Castle 291
Beaumont, Francis 338

Belleforest, François de 2, 4-5, 69-70, 74-7, 79-88, 90-102, 104-8, 110

Bembo, Cardinal Pietro 32, 202

Beroaldo, Filippo 22

Berosus see pseudo-Berosus

Bette, Thomas 24, 339

Bible

1 Samuel 8, 174-5

Old Testament, 174

New Testament, 206

Biondi, Sir Giovanni Francesco 337

Blois, Treaty of see Treaty of Blois

Boccaccio, Giovanni 11

Bochetel, Guillaume 33

Bohemia, Elizabeth of see Stuart, Princess Elizabeth, Countess Palatine of the Rhine

Bohemia, Frederick of see Frederick V Bonnvitius (Bonnuitius, Bonutius), Juda $67-8,76$

Bonnvitius (Bonnuitius, Bonutius), Nicolaus 67-8, 76

Bourbon, Henri de 239

Bourbon, Henri de, Henri IV of France 30, 117, 124, 176, 242-6, 248-9, 267-9, 271-2, 314

Bourbon, Louis XIII 117, 124, 164, 272

Bourbon, Marguerite de Valois see Valois Bourbon, Marguerite de, Queen of France

Bourbon-Montpensier, Charlotte de see Orange-Nassau-Dillenburg, Charlotte de Bourbon-Montpensier, third Princess consort of

Bourdelotius, Johannes 20, 116-17

Brancas, André de, sieur de Villars 242 breath see erotica

Briareus 201

Brooke, William, tenth Baron Cobham 248 
Brutus 232-4, 236-8, 244

Brutus, Junius 176

Burke, Lady Anne Compton,

Countess of Clanricarde and

St. Albans see Compton Burke,

Lady Anne, Countess of Clanricarde and St. Albans

Burke, Richard, fourth Earl of Clanricarde, first Earl of St. Albans 301

Burke, Ulrick, fifth Earl of Clanricarde, second Earl of St. Albans 301

Burton, Robert 330

Burton, William 5, 70-2, 74-85, 87-8, 90-110, 116, 338

\section{Cadmus 76}

Caesar, Gaius Julius 232-8, 249

Calvin 24, 123, 226, 240

Calvinism (Calvinist) 1-3, 18, 24, 116, 121-3, 129, 133, 151, 177, 194, 226, 240-1, 244, 250, 258-9, 272

Camerarius, Joachim, the younger 193

Camotius, Joannes (Ioannes) Baptista 17

Carey, George, second Lord Hunsdon 248

Carey, Henry, first Lord Hunsdon 41

Carey Howard, Lady Katherine, Countess of Nottingham 41

Caro, Annibale 28, 206

Caroline 65, 89, 97, 100, 305

Carr (Car), Lady Frances Howard

Devereux see Howard

Devereux, Lady Frances,

Countess of Essex (subsequently Lady Frances Howard Devereux Carr, Countess of Somerset)

Carr (Car), Robert, Earl of Somerset 274, 301-2

Casaubon, Isaac 17, 181, 268-9
Casca 232-3, 236

Casimir, John (Johann), Count Palatine of Pfalz-Simmern 272

Cassius 232-5

Castiglione, Baldassare 32, 202

Catanaeus, Joannes Maria 13

Catholic League see League, Catholic

Catholic Reformation 1, 127

Catholic, Roman see Church of Rome

Cavaliers 63, 338

Cavendish Stuart, Lady Elizabeth see Stuart, Lady Elizabeth Cavendish, Countess of Lennox

Cavendish Talbot, Lady Mary, Countess of Shrewsbury 290, 298

Cecil, Lady Dorothy Neville, Countess of Exeter see Neville Cecil, Lady Dorothy, Countess of Exeter

Cecil, Robert 248, 250-2

Cecil, Robert, Earl of Salisbury, see Cecil, Robert

Cecil, Thomas 35, 304

Cecil, Thomas, first Earl of Exeter see Cecil, Thomas

Cecil, William, Lord Burghley 35, 121, 226, 232, 242, 282, 304

Cecil de Vere, Lady Anne, Countess of Oxford 121, 282, 294, 304

Cecil Denny, Lady Mary 304

Cecil Hatton (or Newport), Lady Elizabeth 35

Celt (Celts) 165, 171, 175, 179

Celta, Stephano Iunio Bruto 175

Celtophile see Estienne, Henri

Centaurs 131, 193-5

Cervantes Saavedra, Miguel de 20-1, $121,182,279,299-300,330$

Ceyx 184

characterization see rhetoric

Chariton 35

Charlemagne 250, 271-2

Charles I see Stuart, Charles I

Charles V, Holy Roman Emperor 17 
Charles IX see Valois, Charles IX Charles, Prince see Stuart, Charles I

Charles, Prince, see Stuart, Charles II chastity (chaste, chast) see erotica chivalry (chivalric) 11, 20, 46, 122, $127,138,169,180,182,183$, $195,224,278,288-9,294,300$, $311,313,315,336,339$

Christ 262, 272, 338

Christ Church College, Oxford 70 Christ's College, Cambridge 36 Christian 1, 3, 18-19, 24-5, 32, 66, $121,123,127,129,161,170-2$, 194, 202, 216-19, 241, 244, 256, 261-2, 266-8, 271-2, 275, 283-4, 287, 292, 299-300, $309,312-13,315-17,325,330$, 335-6, 338-9

Church of England 268-9

Church of Rome 1, 24-5, 29, 115, 119, $127,139,144,176-7,193,226$, 239, 241-4, 246-7, 250, 253, 255-6, 266-70, 272, 274, 276, 299, 300-1, 314, 339

Church, Gallican 29, 157, 178, 268-9

Cicero (Ciceronian) 18, 23, 59, 67, 77, $83,229,231,233-4,282$

Clary, François de 249

Cleopatra 142, 238, 244-5, 247-9, 253-4

Clèves, Princess Henriette de, Countesse de Nevers 3, 88, 124, 159, 163-5, 272, 314, 337

Clifford, George, third Earl of Cumberland 42, 255

Clifford Sackville Herbert, Lady Anne, Countess of Pembroke, Dorset Clovis 271 and Montgomery 255, 282, 318

Clytemnestra 186

Cobham, Lord see Brooke, William, tenth Baron Cobham

Coccio, Francesco Angelo 66-7

Coldock, Francis 179
Colet, Claude 286

Collège des Lecteurs Royaux 33

Columbani (Columbanius), Raphael 34

comedy see theatre

Comingeois, B. see Belleforest, François de

Commelinus (Commelini), Hieronymus $67-8,70-1,116$

Compton, Sir William, first Earl of Northampton 301

Compton Burke, Lady Anne, Countess of Clanricarde and St. Albans 301

Constantine, Sir William 212

Coriolanus 229-31, 241

Coriolanus see Shakespeare

Cornelia 238

Council of Trent (tridentine) 127, 241, 268

Counter-Reformation 1, 253

Courier de Méré, Paul-Louis 3-5, 34, 38, 48, 52, 206

Coutras, Battle of 241

Crassus 238

Crato von Crafftheim, Johannes 116

Creede, Thomas 70

Cromwell, Oliver 36-7

Cruceius, Hanniball see della Croce, Ludovico Annibale

Crusius (Crucius), Martinus see Kraus, Martin

Cuffe, Henry 34

Cunobelinus see Cymbeline

Cupid 15, 22, 44, 104-5, 170, 201, 223, 277, 311-12

Cymbeline 266

Cymbeline see Shakespeare

d’Angenne, Nicolas, Seigneur de Rambouillet 238

Daphnis and Chloe see Longus

D’Avenant, Sir William 338-9

d'Urfé, Honoré 73 
da Bisticci, Vespasiano 226

Danae 130

Daniel, Samuel 6, 20, 238, 245-6, 248, 255, 327

Day (Daye), Angel 4, 35-6, 38-44, 46-8, 51-63, 79-80, 123, 255, 312,337

Day, John 177

Day (Daye), Richard 177

de Bèze, Théodore 226, 240

de Burgh see Burke

de Clary, François 249

de Dinteville, Jean 29

de Faur, Guy, Seigneur de Pibrac 39

Defence of Poetry see Sidney, Sir Philip de L'Ecluse, Charles (Carolus Clusius) $116,226-7$

de la Torre, Conte Michele, Vescovo di Ceneda 118

de Luján, Pedro 183, 219

de Médicis, Catherine, Queen of France 34, 66, 117-18, 280

de Médicis, Giuliano 117

de Médicis, Marie, Queen of France 117, 314

de Mena, Fernando 118-19

de Montmorency, Philip, Count of Hoorn (Horn) 177, 267

de Mornay, Philippe, Seigneur du Plessis-Marly see Du PlessisMornay, Philippe, Seigneur du Plessis-Marly

de praesenti marriage see erotica de Selve, Georges 29

de Silva, Feliciano 182

de Vere see Vere

Declaration exhibited to the French King 177-8

Declaration of the Clergy of France 268

Delius, Matthäus 115

della Croce, Ludovico Annibale 5, $66-7,69,70,72,74-85,87-8$, 90-2, 94-102, 104-8, 110, 114
Delphic oracle 76, 269

Demosthenes 23

Denny, Sir Anthony 304

Denny, Sir Edward 304

Denny, Lady Mary Cecil see Cecil

Denny, Lady Mary

Denny Hay, Lady Honora, Countess of Carlisle 304

Deukalion 130

Devereux circle 25, 70, 80, 251, 273, 301

Devereux, Lady Frances Howard, Countess of Essex see Howard Devereux, Lady Frances, Countess of Essex (subsequently Lady Frances Howard Devereux Carr, Countess of Somerset)

Devereux, Lady Frances Walsingham Sidney, Countess of Essex see Walsingham Sidney Devereux Burke, Lady Frances, Countess of Clanricard and St. Albans

Devereux, Robert, second Earl of Essex 25, 34, 41-2, 44-6, 121, 198, 242-3, 245-51, 255, 301, 318,323

Devereux, Robert, third Earl of Essex 301

Devereux Dudley, Lady Lettice Knollys, Countess of Essex and of Leicester see Dudley, Lady Lettice Knollys Devereux, Countess of Essex and of Leicester

Devereux Perrot Percy, Lady Dorothy, Countess of Northumberland 323

Devereux Rich, Lady Penelope, Countess of Warwick (subsequently Lady Penelope Devereux Rich Blount, Countess of Devonshire) 250, 286, 301 
Devereux Seymour, Lady Frances, Duchess of Somerset 318

Device 12, 47, 82, 84, 88, 170, 186, 191-3, 195-9, 216, 295, 320, 327, 329-30

di Cosimo, Piero 117

Diana 44, 102, 153, 173, 197-8, 277

Diaz, Juan 182

Digges, Dudley 280

Dio, Cassius 233-4

Dionysus 39-41, 49, 53-4, 62, 130

Dolabella 253

Dolce, Ludovico 66

Doni, Anton Francesco 228

Donne, John 25, 201, 247

Dousa, Janus 68, 226

drama see theatre

Drayton, Michael 338

dream 11, 37, 60-1, 87-90, 93, 95-6, $100,128,137,140-1,156,173$, $199,207,217,253,262,286$, 293, 309-10, 319

Druis 172

du Bartas, Guillaume de Saluste 123, $126,163,243$

Du Coignet, Pierre 269

Du Moulin, Pierre 269

Du Perron, Cardinal Jacques Davy 268-70

Du Plessis-Mornay, Madame Charlotte Arbaleste 177, 242-4

Du Plessis-Mornay, Pierre, Seigneur de Buhy 242

Du Plessis-Mornay, Philippe, Seigneur du Plessis-Marly 172, 175, 177, $217,219,239,241-4$

Dudley circle 13

Dudley, Ambrose, Earl of Warwick 119

Dudley, Lady Lettice Knollys Devereux, Countess of Essex and of Leicester 41, 323

Dudley, Sir Robert, Earl of Leicester 13, 42, 120-1, 169, 172, 192,
$224,227-8,245,250-1,307$,

323

Dürer, Albrecht 17

Dutch Church, London 24-5, 193

Dyer, Sir Edward 45, 192

ecphrasis (ecphrastic) see rhetoric

Edict of Fontainebleau 339

Edict of Nantes 246, 339

Edward IV 16

Egmont, Lamoral, Count of 177, 267

Egmont and Buren, Anne of see Orange-Nassau-Dillenburg, Anne of Egmont and Buren, first Princess consort of

Elector 68, 116, 123, 272

Elizabeth I see Tudor, Elizabeth I

Elizabethan 3, 6, 13, 16, 24-5, 31, 41-2, 44-5, 47, 59, 63, 65-6, $68,70,74,78-9,98,100-1$, $117,124,142,170,174-5$, $225,231-2,243,249,255,306$, 334

Ely House 42, 45

Emathius 171

Emperor (Emperour), Holy Roman see Holy Roman Emperor

Epaminondas 228

Erasmus 24

Ermine 186, 201, 258, 260-1

Eros 37, 39, 82-3, 105,

erotica $64,105,211,323$

breath 202, 207-8, 216, 265, 312

chastity (chaste, chast) $18,32,44$, 63-4, 72-3, 91, 101-2, 106-11, $113,117,153-4,163,184$, 186-7, 197, 199-200, 204-5, 215, 221-2, 247, 257, 260-1, 278, 287, 299, 309, 314 de praesenti marriage 60 heterosexual (heterosexuality) 63-4, 73, 198

homoerotic (homoeroticism) 64, 99, 198, 302 
homosexual (homosexuality) $56-7,63,73,90$

kiss (kissing, kissed) 32, 38-40, 48-9, 51, 57, 60-3, 98-100, 105, 200-3, 207-9, 216, 219, 311

lesbian 55, 198, 203

marriage (marry, married) 6, 18, 27, 29-30, 35, 45-7, 49, 53, 55, 59-63, 65, 87, 89-90, 92, 102, 104, 106-7, 109, 112, 120-1, 123-4, 130, 139-40, 144, 147, $150,155,157,160-2,179,182$, 185, 187-9, 192, 195, 199-206, 213-18, 221-4, 239, 252, 255, 257, 263-4, 266, 268, 272, 274-5, 278-83, 286, 290-4, 296-301, 304-5, 308-9, 311, 313-14, 316-18, 320-4, 326-9, 332,335

orgasm 50, 99, 202, 210

sex $21,32,44,51,64,72,90,92$, 95-8, 102-4, 109-11, 140, 202, 205-6, 210, 217, 221, 223-4, 315

soul (soule) $32,73,88,104,128$, $185,201-3,206,208,210,216$, 218, 284-5, 311, 327 virgin (virgine, virginity, virginitie) $41,44-5,48,51,60-2,81$, 88, 90-2, 96, 98, 100-2, 104, $106-9,137-8,140,159-60$, $198,200,264,278$

Essex see Devereux

Estienne, François 259

Estienne, Henri 32-3, 65, 68, 164, 175-6, 206-7, 259, 280

ethopoeia see rhetoric euphemism see rhetoric

Euripides (Euripidean) 33, 134-5

Europa 66, 76-8, 80-1, 90, 93, 95, 109, $171,183-4$

Fairy Queen (The Faerie Queene) see Spenser, Edmund
Ferdinand II, Holy Roman Emperor 276

Fitton, Mary 291

Fitzgerald, Lady Frances Howard, Countess of Kildare see Howard Fitzgerald, Lady Frances,

Countess of Kildare

Fitzgerald, Henry, twelfth Earl of Kildare 45

Fletcher, John 299, 338

Fletcher, Phineas 38

Florio, John 20

Fondulo, Jerome 28

Fontainebleau, Edict of see Edict of Fontainebleau

France, Marguerite de see Valois Bourbon, Marguerite de, Queen of France

France, Parliament of 178

François Hercule, Duc d'Anjou see Valois, François Hercule, Duc d'Anjou

François I see Valois, François I François II see Valois, François II

Fraunce, Abraham 5, 122, 133

Frederick IV, Count Palatine of the Rhine 272

Frederick V of Bohemia, Count Palatine of the Rhine 123-4, 272, 276, 305

Fulgentius 71

Galileo 72

Gallic 25, 127, 163, 165

Gallican (Gallicanism) 29, 157, 178, 250, 268-9

Gallus, Comerus 171

Gamage family 25, 279

Gamage Sidney, Lady Barbara, Countess of Leicester 41, 45, 73, 306-7

Gambara, Laurentius 68

garden $12,15,39,53-6,79-80,93-5$, $192,209,223,263,320$ 
Gargantua 193

Garnier, Robert 6, 238-40, 244-6, 248

Gawdy, Bassingbourne 41

Gawdy, Philip 41-2, 47

Gayton, Edward 21, 338-9

gesture see rhetoric

Ghini, Leonardo 118

Gifford, Dr. William 46

Gigli, Silvestro, Decano di Lucca 66

Giolito De’ Ferrari, Gabriele 118

Giovio, Paolo 327

Glorious Revolution 339

Gloucester, Richard Duke of see Richard III

God 37, 133, 136, 144, 151, 156, 174-8, 201, 205, 217-18, 240-1, 243, 249, 256, 338

Gohory, Jacques 127, 336-7

Golden Fleece, Order of the 177, 193

Gonzaga, Ludovico (Louis de

Gonzague), Duc de Nevers 246

Goodale, John 174

Goodman, Christopher 175

Gorgias 103, 259

Gorgon 131, 195

Gough, John 105, 122, 126-7

Goulart, Simon, Senlisien 18, 123, 163, 240-1, 258-9, 261-2

Grammaticus, Aelfric 123

Greco-Roman 1-5, 10, 13, 16, 20-2, $24,33,36,39-40,58,62,65$, $74,76,78,88,92,110,113,160$, $164-5,182,194,198-9,202$, 224-6, 232, 254, 270, 278, 285, $299,331,333,336,339$

Greek Orthodox 115

Greene, Robert 66, 255-7, 262-3

Gresham, Thomas 182

Greville, Fulke 6, 20, 25, 45, 178-80, 192, 212, 237-8, 241, 246-50, 305-6

Grey Seymour, Lady Katherine, Countess of Hertford 318

Groulleau, Estienne 21, 31, 117
Gruter, Jan see Gruytere, Jan de (Janus Gruterus)

Gruytere, Jan de (Janus Gruterus) 68, 116

Guevara, Antonio de 227-8, 255

Guise, Henri, third Duc de see Guise, Henri de Lorraine, third Duc de

Guise, Henri de Lorraine, third Duc de 30, 241, 243-4

Guise, Louis II de Lorraine, second Cardinal de 241

Gunpowder Plot 253, 268

Gynaecia 169

H., A. 73

Habsburg 270, 276, 299

Hacket, Thomas 182

Hannibal 266-7

Harington, Sir John 138

Harrington Rudyerd, Lady Mary 320

Harrison, William 172

Harvey, Gabriel 176

Hatton, Sir Christopher 35, 42, 45

Hatton (or Newport), Lady Elizabeth see Cecil Hatton (or Newport), Lady Elizabeth

Hatton (or Newport), Sir William 35, 42,61

Hay, Lady Honora Denny, Countess of Carlisle see Denny Hay, Lady Honora, Countess of Carlisle

Hay, James, first Earl of Carlisle 304-5 Hay, Lady Lucy Percy, Countess of Carlisle see Percy Hay, Lady Lucy, Countess of Carlisle Hayward (Hayvvard), James 337 Heliodorus 1-2, 4-6, 10, 12, 15-16, 19-20, 22, 24-5, 27-34, 65-8, 71-3, 77, 102, 110, 111-65, 168-9, 174, 179, 181, 183-4, 187-8, 190, 195-6, 199-200, 203-5, 209-10, 217-19, 222-3, $229,231,254,260,265,270$, 
283-5, 299, 316, 321, 326, 330, 336-7

An Ethiopian Story 10, 11, 15, 20, $22,29,31,33,72-3,103,110$, 111-65, 183-5, 189, 193, 199, 203-4, 208, 219, 222-3, 229, 263, 266-7, 295

Hellen, son of Deukalion 130

Hellenes 130

Hellenism 24, 139

Heneage, Thomas 35

Henriette-Marie (Henrietta Maria),

Queen 3, 124, 159, 163-5, 272, 314

Henri II 29-30, 117, 227, 336

Henri III see Valois, Henri (Henry) de

Henri III of Navarre see Bourbon, Henri de, Henri IV of France

Henry $V$ see Shakespeare

Henry VIII see Tudor, Henry VIII

Henslowe, Philip 243

Herberay des Essarts, Nicolas de 299

Herbert family 6, 25, 273, 296, 298, 334

Herbert, Anne 291-2

Herbert, Henry, second Earl of Pembroke 121, 240, 287, 291, 298, 307

Herbert, Lady Anne Parr, Countess of Pembroke see Parr Herbert, Lady Anne, Countess of Pembroke

Herbert, Lady Anne Clifford Sackville, Countess of Pembroke, Dorset and Montgomery see Clifford Sackville Herbert, Lady Anne, Countess of Pembroke, Dorset and Montgomery

Herbert, Lady Catherine Talbot, Countess of Pembroke see Talbot Herbert, Lady Catherine, Countess of Pembroke

Herbert, Lady Mary Sidney, Countess of Pembroke see Sidney Herbert, Lady Mary, Countess of Pembroke

Herbert, Lady Mary Talbot, Countess of Pembroke 290, 298

Herbert, Philip, first Earl of Montgomery and fourth Earl of Pembroke 122, 251-2, 282, 287, 294, 299, 329,

Herbert, Sir Edward 314

Herbert, William, third Earl of Pembroke 7, 20, 252, 273-4, 276, 280, 289-90, 295-8, 302-3, 318

Herbert Talbot, Lady Anne 298

Hercules 196

Hérembert, Jean, Sieur de la Rivière 69

Hervet (Hervetus), Gentian 13

Herwagen, Johannes 67, 114, 116

heterosexual (heterosexuality) see erotica

Himenaeus see Hymen

Hippolytus 92-3

Hobart (Hubbard), Lady Philip (Philippa) Sidney see Sidney Hobart (Hubbard), Lady Philip (Philippa)

Hobart (Hubbard), Sir John 306, 309-10

Hoby, Sir Thomas 32

Hodges, Anthony 5, 36, 72-9, 81-2, 84-93, 95-7, 100-8, 110, 306, 338

Holbein, Hans 29

Holinshed, Raphael 165, 172

Holland, Philemon 30, 259

Holy Roman Emperor 17, 30, 123, 192, 253, 276, 299, 308, 319, $321,326,331$

Holy Roman Empire 17, 270, 275, 321,328

Homer (Homeric) 21, 113, 131, 134-5, 146, 190, 195 
homoerotic (homoeroticism) see erotica

homosexual (homosexuality) see erotica

Hoorn (Horn), Philip de Montmorency, Count of see de Montmorency, Philip, Count of Hoorn (Horn)

Hoskyns (Hoskins), John 181-3

Howard family 301

Howard, Charles, Baron Howard of

Effingham and first Earl of

Nottingham 41-2, 44-5, 47, 56, 61,123

Howard, Henry, Earl of Northampton 250

Howard, Henry, Earl of Surrey 250

Howard, Lady Katherine Carey,

Countess of Nottingham see

Carey Howard, Lady Katherine,

Countess of Nottingham

Howard, Lady Katherine Knevet

Rich see Rich Howard, Lady

Katherine Knevet, Countess of

Suffolk

Howard, Sir Thomas, first Earl of

Suffolk (1561-1626) 301

Howard, Sir Thomas, fourth Duke of

Norfolk (1538-72) 121, 301

Howard Devereux, Lady Frances,

Countess of Essex (subse-

quently Lady Frances Howard

Devereux Carr, Countess of

Somerset) 301

Howard Fitzgerald, Lady Frances,

Countess of Kildare 45

Howard Leveson, Lady Margaret 47

Howard Southwell, Lady Elizabeth 45

Howell, James 306

Huguenot (Huguenots) 24-5, 30, 32,

43, 46, 117, 119-20, 123, 164,

$172,206,211,227,239-46,271$,

280,314

Hunt, Henry 211
Hurtado de Mendoza, Don Diego 66

Hussite Protestantism 256

Hymen 62, 137-8, 301

hyperbole see rhetoric

impresa (imprese) 12, 89, 170, 191,

216-17, 327

Interest theory (interest) 119, 173-4,

$178,181,189,211-13,215,222$,

224-5, 250, 258, 287-9, 296,

$300,322-4,327,339$

Isis $137-8,142$

Istr 171

Ivan IV, the Terrible 177

Jacobean 6, 20, 24, 74, 124, 142, 228, $249,256,258,261,263,266$, 275, 279, 304, 306, 319, 334

James VI and I see Stuart, James VI and I

Janus 171

Japhet 165

Jemmingen, Battle of 177

Jesuit 74, 116, 253, 269

Jewish uprising 234

Jonson (Iohnson), Ben 181, 249, 274, $301,334,338$

Jove 173

Jubal 171

Julius Ceasar see Shakespeare

Jungermann, Gottfried 36, 38

Juno 153

Jupiter 248

kiss (kissing, kissed) see erotica

Knevet Rich Howard, Lady Katherine, Countess of Suffolk see Rich Howard, Lady Katherine Knevet, Countess of Suffolk

Knollys, William, first Earl of Banbury 251

Knollys Devereux Dudley, Lady

Lettice, Countess of Essex and of Leicester see Dudley, Lady 
Lettice Knollys Devereux, Countess of Essex and of

Leicester

Kraus, Martin (Martinus Crusius) $71-2,116$

Kyd, Thomas 24

lesbian see erotica

L'Isle (Lisle), William 5, 122-6, 132-3, $136,138,140-1,143-4,147-9$, $151-6,159,161-5,338$

The Faire Aethiopian 5, 123-4, 126, $151,153,162-3$

La Tour d'Auvergne, Henri de, Viscount of Turenne 242

Languet, Hubert 116, 168-9, 171, $173-5,177-9,212-13,239$, 272,279

Lant, Thomas 46

Lapiths 131, 193-5

Laura 170

Le Grys, Sir Robert 306

League, Catholic 242

League, Holy 178

League, Protestant 178-9, 214, 276, 287

Lee, Sir Henry 42, 45, 191-3

Leicester, Earl of see Dudley, Sir Robert and Sidney, Sir Robert

Lemaire de Belges, Jean 171

Lepidus 234, 253

Leukippe and Kleitophon see Achilles Tatius

Leveson, Lady Margaret Howard see Howard Leveson, Lady Margaret

Leveson, Sir Richard 47

l'Huillier, Pierre 69

Lingelshemio, Georgio Michaeli 116 Lisle, Lady see Gamage Sidney, Lady Barbara, Countess of Leicester Lives of the Sophists see Philostratus Lloyd, David 339

London, Treaty of see Treaty of
London

Longis, Groulleau and Sertenas, printer-publishers 28-9, 31, $117-18,128$

Longus $1-2,4,10,12,15-16,24,26$, 27-63, 64, 68, 76, 90, 168, 184, 198, 200, 206-7, 260, 284, 334 Daphnis and Chloe 4-5, 10-11, 15, 27-63, 64, 66, 73, 76, 79-80, 103, 111, 123, 171, 199, 206-7, $215,222,255,263,281,337$

Lord Chamberlain's Men 248

Lord Strange's Men 243

Lorich (Lorichius, Lorichij), Reinhard 13

Louis XIII 17, 124, 164, 272

Lovelace, Richard 73, 89

Love's Labours Lost see Shakespeare

Love's Victory see Sidney Wroth

Loyseleur, Pierre, Sieur de Villiers 178

Lucian (Lucianic) 14, 176

Luther, Martin 115

Lutheran 13, 68, 71

Lycurgus 189, 240

Lyly, John 31

Macbeth see Shakespeare

Machiavelli, Niccolò di Bernardo dei 211,319

Magus 172

Malory, Sir Thomas

Morte D'Arthur (Mort Darthure)

12, 122

Manners, Roger, fifth Earl of Rutland 269

Manners, Sir George, seventh Earl of Rutland 306

Manutius, Aldus 14

Manzini, G. B. 28

Maplet, John 158

Maria Anna, Infanta of Spain 30, 124

Marian exile 175

Marlowe, Christopher 24, 196, 243-4, 280 
marriage (marry, married) see erotica Martin Marprelate 43

Mary I see Tudor, Mary I

Mary, Queen of Scots see Stuart, Mary, Queen of Scots

masque see theatre

Mathias, Holy Roman Emperor 276

Maximilian II, Holy Roman Emperor 30

May, Thomas 306

Measure for Measure see Shakespeare medieval 11, 22, 56, 67, 127, 300

Medusa 130

Melanchthon (Melancton), Philip 115-16, 120, 168, 174, 176

Memnon 130, 163

Mesa 171

Messala 232, 234

metaphor see rhetoric

metonym (metonymic) see rhetoric

Michelangelo 265

Midas 169

Milton, John 36

monarchomachia (monarchomachist) $89,169,174-5,177,179,183$, $185,195,212,214,224-5,236$, $241,246,249,252,256,258$, 261, 268-9, 285, 306, 314, 316, $321,334,336,338-9$

monarchy $1,3,13-14,29,36,130$, $161,168,174,178,213-14,225$, 237, 247-8, 257, 263, 271-2, $319,322,326,330,339$

Montaigne, Michel de 33

Montemayor, Jorge de 20, 181, 284

Morand, Thomas, Duc du MesnilGarnier 117

Mornay, Philippe de, Seigneur du Plessis-Marly see Du PlessisMornay, Philippe, Seigneur du Plessis-Marly

Mort Darthure (Morte D'Arthur) see Malory, Sir Thomas

Munday, Antony (M[unday],
Muslim 339

A[ntony]) 294, 299

Nantes, Edict of see Edict of Nantes

Nassau, Elisabeth (Elizabeth) of see Orange-Nassau-Dillenburg de La Tour d'Auvergne, Elisabeth (Elizabeth), Princess of Nassau, third Viscountess of Turenne

Nassau, Marie of see Orange-NassauDillenburg of HohenloheNeuenstein, Marie, Princess of National (nationalism, nationalist) $2,17,24-5,30,52,65,69,78$, $118,123,125,127,159,161-2$, $164-5,168,171,178,192,211$, 224-5, 231, 234, 237-8, 249, 256, 259, 268, 324, 334, 336, 339

Navarre, Henri III of see Bourbon, Henri de, Henri IV of France

Negri, Stefano (Stephani Nigri) 14

neoplatonism (neoplatonic, neoplatonist) $17,21,32,67,88$, 110-11, 202

Neoptolemos 130, 193

Nevers, Princess Henriette de Clèves, Countesse de see Clèves, Princess Henriette de, Countesse de Nevers

Nevers, Ludovico Gonzaga (Louis de Gonzague), Duc de see Gonzaga, Ludovico (Louis de Gonzague), Duc de Nevers

Neville, John, fourth Baron Latimer 304

Neville Cecil, Lady Dorothy, Countess of Exeter 304-5

New Testament see Bible

Newport, Sir William see Hatton (or Newport), Sir William

Nichols, John 306

nightmare see dream 
Noah 5, 165, 168, 171-2

Norman 25, 124, 224, 242, 279

North, Roger, second Baron North 227-8

North, Thomas 6, 30, 142, 169, $225-32,234,236-7,254-5,270$

novel 1, 3-5, 11-12, 15, 17-20, 22, 27-9, 35, 37-8, 51, 53-4, 62-3, 65-7, 71-2, 77-8, 81-2, 85, 88, 93-5, 106-11, 127-8, 130-1, 140-2, 186, 195, 215, 223, 235, $260,287,297,335,339$

nymph (nymphe) 15, 28, 37-8, 40, 43-4, 61, 191, 197

O.E.D. $11-12,38,49,51,70,137,158$, 231, 280, 282, 289, 298

Obsopoeus (Opsopaeus), Vincentus $71,114-17,127$

Octavia 247

Octavius 233-4, 237-8, 244, 246, 248-50, 253 see also Augustus

Odysseus 131

Old Testament see Bible

Omphale 196

Oporin, Johann 115

Oracle 11, 76, 169, 184, 202, 204-5, 221, 257-8, 262-3, 265, 267

Orange, Prince of see Orange-NassauDillenburg, Willem I, Prince of

Orange-Nassau-Dillenburg, Anne of Egmont and Buren, first Princess consort of 192

Orange-Nassau-Dillenburg, Charlotte de Bourbon-Montpensier, third Princess consort of 192

Orange-Nassau-Dillenburg, Louise Juliana of, Princess of NassauOrange, Countess Palatine of the Rhine, mother of Frederick $\mathrm{V}$, Count Palatine of the Rhine 272

Orange-Nassau-Dillenburg, Willem I, Prince of (William 'The Silent')
$177-8,192-3,226$

Orange-Nassau-Dillenburg de La

Tour d'Auvergne, Elisabeth

(Elizabeth), Princess of Nassau, third Viscountess of Turenne 192

Orange-Nassau-Dillenburg of Hohenlohe-Neuenstein, Marie

of, Princess of Nassau 192, 222

Orestes 130

orgasm see erotica

Orsini, Fulvio 28, 34, 65, 206

Ovid 121, 130, 184-5, 265, 317

Oxford see Vere

Oxinden, Henry 306

painting $12,14,27-8,54,76-8,82,90$, 93-4, 103, 109, 117, 130, 132, 169, 184, 194

Palatine, Elector 116, 123, 272

Pallavicino, Petro Francisco (Pierfrancesco) 67

Pamphilia see Sidney Wroth

Pan 37, 39-40, 43, 54, 58, 62, 109

papal see Pope

parable see rhetoric

paradox see rhetoric

Parma, Duchess of 177

Parr Herbert, Lady Anne, Countess of Pembroke 298

Parr Tudor, Queen Katherine 298

Parthenius 34, 68

Paulet, Sir Hugh 119-20

Paull, Andreas 116

Paynell, Thomas 182, 196, 219, 277

Peele, George 46

Pembroke see Herbert

Percy, Henry, ninth Earl of Northumberland 305, 322

Percy Hay, Lady Lucy, Countess of Carlisle 305

Percy Sidney, Lady Dorothy, Countess of Leicester 305, 322-3

periphrasis (periphrastic) see rhetoric 
Perseus 22, 111-12, 117, 130, 162-3, 195

Petrarch (Petrarca), Francesco 170

Philathenian 23

Philhellene 1, 3, 5-6, 13, 18, 22-3, $25,30,65,68,70-1,80,98$, $112,114-16,119,121-4,127$, $129,131,134,147,154,161-2$, 164-5, 174, 196, 205, 211, 214, 224-8, 231-2, 234, 236, 239, 244, 246, 248-9, 251-3, 255, 258-9, 262, 266, 270-2, 274, 276, 279-80, 283, 300, 304, 307, 313-15, 317-18, 320, 324, 327, 330-1, 334-5, 338-9

Philhellene Protestant 3, 5-6, 13, 30, $65,68,70-1,80,98,115-16$, $119,122-4,162,165,174,196$, 205, 214, 224-8, 231-2, 236, $239,246,248-9,251-3,255$, 258-9, 262, 266, 270-2, 274, 276, 279-80, 283, 300, 304, 307, 313-15, 317-18, 320, 324, 327, 330-1, 334-5, 338-9

Philip II of Spain 177, 193, 226, 237, $241,244,249,271$

Philip III of Spain 253, 256

Philip IV of Spain 124

Philip of Macedon 228

Philisides 170-4, 178-9, 190-1, 193, 200-1, 204, 206-7, 210, 305

Philomela 103

Philostratii 22

Philostratus, Flavius 233

Lives of the Sophists 233

Philostratus, the elder 4, 12, 14-15, 265

Philostratus, the younger 15

Photius 65-6, 74

Pibrac see de Faur, Guy, Seigneur de Pibrac

Pico della Mirandula see Picus, John (Pico della Mirandula)

Picus, John (Pico della Mirandula) 17
Picus, John Francis 17

Pirkheimer (Pirkeymheru[m]), Bilibald (Willibald) 17

plain style (plain, plainness, playn, playne) see rhetoric

Plantin, Christopher 226

Plato (Platonic, Platonist) 1, 74-5, 94, $300,311,315$

play see theatre

Pléiade 337

Plutarch (Plutarchan) 2, 4-6, 15-18, 21-4, 29-31, 33-4, 86, 110-11, $118,121,127-9,142,152-3$, 169, 185-6, 211, 224-32, 238, 240, 245, 247-8, 254, 258-9, $262,270,287,326,332,334$

Pluto 248

Polybius 230

polyptoton see rhetoric

Pompey (Pompeyist) 18, 232-4, 236-8, 249

Ponsonby, William 179

Pope (papal) 25, 172, 179, 243-4, 267-71, 276

Pope Clement VIII 267

Pope Paul V 267

Pope Sixtus V 271

Portia see Shakespeare, The Merchant of Venice

Portia, wife of Brutus 238

Poseidon 132, 147

Postel, Guillaume 271, 339

Presbyterian 211

Priam, King of Troy 130

prosopopoeia see rhetoric

Protestant 1-3, 5-6, 13, 17, 24-6, $30,45,65,67-8,70-2,80,98$, 114-16, 119, 121-4, 126, 162, $165,168-71,174,178-9,193$, $196,205,212,214,220,224-8$, 231-2, 236, 239-41, 244-53, 255-6, 258-9, 262, 266-76, $278-80,283,287-8,299-300$, 304, 307, 313-15, 317-20, 
$322-4,326-7,330-1,334-6$, 338-9

Protestant League see League, Protestant

Prusa, Dion of 233

Prynne, William 176

Pseudo-Berosus 164, 168, 171-3

Publicola 241

Puritan (Puritanism) 6, 36, 43, 63, 72-3, 122, 125, 259, 274, 338

Pynson, Richard 13

Pythian Apollo 112, 130, 152

Pythian Games 130

Rabelais, François 193

Rainolde, R. see Reynolds, Richard

Ralegh, Sir Walter 248, 252

Ramist 330

Randolph (Rondolph), Thomas 338

Reformation 1, 27, 174, 253, 264, 269, 326

Reformation, Catholic see Catholic Reformation

Reformed Church 24, 68, 177, 334

Renaissance 1, 2, 4, 10-12, 16-22, 24, 28-9, 31-3, 37, 45, 50, 55-6, $64-5,67,71,74-6,80,82-3$, $88,90-1,110,114-17,119,127$, $129,132,142,149,152,154$, $158,161-2,168,170,174,181$, $186,194,201-2,224,226,232$, 253-4, 260, 266, 270, 284-5, 300, 315, 333-4

republic (republican) 1-3, 24-5, 36-7, 114-15, 117, 129, 147, 164, 177, $213,232,249,252,306$

Restoration 336, 339

Retz, Countesse de 337

Rex Christianissimus 271

Reyner, Edward 338

Reynoldes, R. see Reynolds, Richard Reynolds (Reynoldes, Rainolde),

Richard 13-14, 16

rhetoric (rethoricke, rhetorical) 1-6,
$10-15,17,21-3,25,31,33-4$, $36,38-9,62,64-5,70,74-5$, $79,80,82,84-8,90,93-7,100$, 102, 107-10, 112-14, 118-19, $131,133,138,146,152,158$, $168,179,182-3,185,203-7$, 212, 218-20, 226, 231-6, 244, 248, 257, 258, 260-1, 264, 266, $270,275,281,283,286,296$, $305,311,315,321,331-4,336$, 338-9

allegory (allegorical, allegorically, allegorise, allegorised) 1, 4, 6, $12,14,16,18,21-2,25,27,47$, $62,64-5,67,71-2,74,84,89$, $106,110,112,115,117,121$, $127,129,160-2,171,174,186$, $188,211,225,238,240,244$, 247-8, 253, 256, 273, 275-6, 278-9, 285-90, 293-8, 300, 303-4, 306, 309-10, 313, 316, $318,321,324-6,329,332-3$, 337-9

aposiopesis 218

Attic (Atticist) 15, 22

characterization $4,15-16,31,62$, $83-5,98,110-11,121,127,129$, $152,211,215,238,258,270$, $273,287,303,321,326,336$ ecphrasis (ecphrastic) 4, 12, 14-5, 27-8, 38-9, 44, 53-4, 63, 65-6, 76-8, 80-2, 84, 87, 92-3, 98, $100,103,110,131-2,156,191$, 194, 216, 224, 254, 265, 282, $289,312,327$

ethopoeia 14-15, 211, 220

euphemism 51, 141, 155 gesture 46-7, 82, 208, 235, 277 hyperbole 253,311 metaphor $14,45,50,52,58,73,80$, 96, 99, 105, 110, 131-4, 137, 143, 161, 183, 194, 210, 218, 229-31, 234-5, 246-7, 253, $255,257-8,265,289,293,311$ 
metonym (metonymic) 163, 231, 236, 336, 338-9

parable 114, 144, 152, 159, 162, $178,184,188,215,224,266$, $317,337,338$

paradox $15,50,59,64,70,78,86$, $93,110,132,184,198,218,260$ periphrasis (periphrastic) 131, 258, 282

plain style (plain, plainness, playn, playne) 2, 6, 18, 22, 36-7, 51, $67,77-8,84,119,128,154$, 185-6, 194, 220, 228-9, 234, 236, 243-4, 247, 264, 267, 270, $315-16,318,328,332-3,337$ polyptoton 105 prosopopoeia $14,17,87,211,220$, 254

'royal' style (style royal) 31,33 , $36-7,69,118,176,224,229,243$, $250,256-8,262,267,270,332$ zeugma 131, 155

Rich, Lady Penelope Devereux see Devereux Rich, Lady Penelope, Countess of Warwick

Rich, Richard 301

Rich, Robert, second Baron Rich 300

Rich, Robert, third Baron Rich, first Earl of Warwick 301

Rich, Robert, fourth Baron Rich, second Earl of Warwick 301

Rich Howard, Lady Katherine Knevet, Countess of Suffolk 301

Rich Wroth, Lady Margaret 301

Rich Wroth, Lady Mary see Sidney Wroth, Lady Mary

Richard III 14, 16

Ridolfi, Niccolo 66

Rogers, Daniel 68

Rohan, Henri, Duc de 211

Roman 2, 6, 10, 17-18, 23, 112, 161, $165,226,230,232-4,237-8$, 249, 267, 275-6, 328, 336

Roman Empire 10, 18, 112, 161,
232-4, 238, 249, 267, 275, 328, 336

Romantic (Romanticism) 3, 11, 28, $87,266,278,281,284,321,327$, $334,336,339$

Rome, Church of see Church of Rome Roquemaure (Rochemaure), Jacques de 69

Rous, Francis 73

'royal' style (style royal) see rhetoric

Rubens, Sir Peter Paul 80

Rudolf II, Holy Roman Emperor 192, 256

Rudyerd, Sir Benjamin 313, 320

Rudyerd, Lady Mary Harrington see Harrington Rudyerd, Lady Mary

Sackville Seymour, Lady Ann, Viscountess Beauchamp 318

Saint Agathan 315

Saint Calliopa 315

Saint Febronia 315

Saint Macra 315

Saint Reparata 315

Saint-Corneille de Compiègne, Abbot of 29,227 see also Amyot, Jaques

Samothes (Samotus) 164-5, 168, $171-2,175,271$

Samuel see Bible

Sanford, Hugh 295, 297-8

Sanford (Sandford), James 5, 119-20

Sannazaro, Jacopo 20, 181

Sarron 172

Satyr 43, 46, 54

scene see theatre

Schottus, Andreas 74

Scipio 226-7, 238

Scots, Mary, Queen of see Stuart, Mary, Queen of Scots

Second Sophistic 10, 12, 15, 19, 22, 31,161

Selene 90

Semele 54 
Seneca (Senecan) 33, 92-3, 194, 219, 238, 243, 254, 264

Sertenas, Vincent 28, 31, 118

sex see erotica

Seymour, Lady Ann Sackville,

Viscountess Beauchamp see

Sackville Seymour, Lady Ann,

Viscountess Beauchamp

Seymour, Lady Arabella Stuart,

Duchess of Somerset see Stuart

Seymour, Lady Arabella,

Duchess of Somerset

Seymour, Edward, first Earl of Hertford 318

Seymour, Edward, Viscount Beauchamp (1561-1612) 318

Seymour, Edward, Viscount Beauchamp (1587-1618) 318

Seymour, Lady Frances Devereux, Duchess of Somerset see Devereux Seymour, Lady Frances, Duchess of Somerset

Seymour, Lady Katherine Grey, Countess of Hertford see Grey Seymour, Lady Katherine, Countess of Hertford

Seymour, William, first Marquess of Hertford and second Duke of Somerset 318

Shakespeare, William 1-3, 5-6, 10-13, 15, 19-20, 25, 44, 49, 56, 70, $89,114,122,135,156,196,210$, 224, 225-70, 283, 306, 311, 328, 334, 336, 338

All's Well that Ends Well 135, 210

Antony and Cleopatra 6, 226, 238, 244, 249-50, 253-4, 258

As You Like It 57, 248

Coriolanus 6, 226, 228-32

Cymbeline 6, 57, 89, 156, 266-7, 269, 275

Henry V 49, 226, 248

Julius Caesar 6, 226, 232-3, 236-8, 243, 246, 248-9, 258, 266
Love's Labours Lost 246

Macbeth 253

Measure for Measure 135, 210

The Merchant of Venice (Portia) 261

The Winter's Tale 6, 11, 20, 89, 255-6, 258, 260, 262-3, 265

Sicily, Queen of see Tudor, Mary I

Sidney family 274, 321

Sidney, Algernon 174, 211, 253

Sidney, Lady Barbara Gamage,

Countess of Leicester see

Gamage Sidney, Lady Barbara,

Countess of Leicester

Sidney, Lady Dorothy Percy, Countess

of Leicester see Percy Sidney,

Lady Dorothy, Countess of

Leicester

Sidney, Lady Frances Walsingham see

Walsingham Sidney Devereux

Burke, Lady Frances, Countess

of Clanricard and St. Albans

Sidney, Sir Henry 175

Sidney, Sir Philip 1-3, 5-6, 10, 12, 15, $19-20,25,30,32,35,43-6,52$, $54,68,72-4,78-80,86,88-9$, $94-5,101,113-16,119,122$, $131,135,146,153,168-224$, $225,227-8,234,237-8,244-6$, 248, 250, 254-5, 257-62, 272, 279, 282-3, 286-7, 294, 300-1, $305,319,330,333-4,337-8$

Arcadia 3, 5, 12, 19-20, 43-4, 52, $54-5,69,72-3,78-80,86,88$, $94-5,113,124,131,135-6,162$, $165,168-224,225,237,246-7$, $250,255,257,260,275,282$, 294-5, 300, 305-6, 319, 327, 334, 337-8

Astrophil and Stella 170, 192, 286

Defence of Poetry 20, 72, 164, 193, 225, 259, 262, 337

Sidney, Sir Robert, Viscount Lisle and first Earl of Leicester 41, 
$68,168-9,174,245,249-52$, 272-3, 275-6, 279, 291, 293-4, 296, 301, 306, 322, 338

Sidney, Sir Robert, second Earl of Leicester 274, 305, 313, 322

Sidney, Sir William (d. 1612) 273, 313 Sidney Herbert, Lady Mary, Countess of Pembroke 6, 20, 30, 55, 122, 217, 238-41, 244-6, 248, 252, 276, 286-8, 294, 298, 307, 310, $319,326,333$

Sidney Hobart (Hubbard), Lady Philip (Philippa) 306

Sidney Wroth (Wroath), Lady Mary $1-3,5-6,10-12,15,19-20$, $27,92,111,114,122,174,214$, 247, 251-2, 261, 270, 271-335, 337-8

Love's Victory 303, 305

Pamphilia to Amphilanthus 276

Urania 6, 11, 20-1, 27, 92, 184, $186,214,247,257,261,270$, 271-335, 337, 339

Sigismund II Augustus, King of Poland 115-16

Sigismund, John, Elector of Brandenburg 68

Sign 338

Silvius, Guillaume (Willem) 226-7

Socrates 10

Sophist (Sophistic) 11-12, 14-15, 19, 21-3, 31, 67, 73-4, 110, 112, $114,157,161,183,185,187$, $221,233,327,332-4,338$

Sophistic see Sophist soul (soule) see erotica

Southampton see Wriothesley, Henry, third Earl of Southampton

Southwell, Lady Elizabeth Howard see Howard Southwell, Lady Elizabeth

Southwell, Sir Robert 45

Spain, Infanta Maria Anna of see Maria Anna, Infanta of Spain
Speed, John 165

Spenser, Edmund 72, 89, 138, 154, 194, 196, 246, 275, 288, 330, 337

Squire, Edward 109

stage see theatre

St. Bartholomew's Day 30, 239

St. Paul's Cathedral 46

Stanley, Ferdinando, Lord Strange, fifth Earl of Derby 243

Stationers' Register 43, 244, 245, 275

Steenwyck, siege of 245

Stoer, Jacob 240

stoic (stoicism) 3, 22, 25, 64, 120, 178, 184, 193-4, 197, 203, 205, 217, 219, 241, 244, 260-1, 265, 275, $283,285,289,315,317$

Stonard, John, of Loughton 300

Stonard Wroth, Lady Susan 300

Stradling 25

Strato 233

Stuart, Charles I 3, 25, 36, 74, 89, 114 , $123-5,127,136,159,162-3$, $165,175,252,257,272,314$, 339

Stuart, Charles II 163

Stuart, Charles, fifth Earl of Lennox 298

Stuart, Princess Elizabeth, Countess Palatine of the Rhine 123, 272

Stuart, Lady Elizabeth Cavendish, Countess of Lennox 298

Stuart, James VI and I 6, 25, 123, 175, 220, 249-53, 256, 259, 263, 263, 267-9, 272, 274-7, 298, 304, 331,333

Stuart, Mary, Queen of Scots 29, 257, 301

Stuart Seymour, Lady Arabella, Duchess of Somerset 298, 318

Stucki, Johannes Wilhelm 250, 271-2

Studley, John 33

style $2,3-4,6,15,18,22-3,29,31$, $33-4,37,43,59,65-6,69,75$, 
$83,93,112-14,118-19,125$, $128,136,168,173,176-7$,

181-2, 186, 194, 206, 214, 218, 220, 224-5, 228-9, 231-8, 243-4, 247, 250, 254-8, 261-2, 266-7, 270-1, 275, 279, 283, $315-16,318,332-4,337-8$ see also rhetoric succession 111, 157-9, 162, 181, 213-14, 238, 250, 252, 267, 283, $286,316-19,321-2,326$

Suda 66

symbol (symbolic) 6, 12, 25, 42, 45, 53-5, 78, 83, 87-9, 95-6, 100-1, $103,106,124,159,162,183-4$, 186, 192-3, 195-9, 216, 236, 258, 263-5, 267, 284, 289, 293, 296, 306, 317, 327, 329, 338

Syrinx 40, 108

Talbot, Lady Anne Herbert see Herbert Talbot, Lady Anne Talbot, Francis, Lord Talbot 298 Talbot, Gilbert, seventh Earl of Shrewsbury 290, 298

Talbot, Lady Mary Cavendish, Countess of Shrewsbury see Cavendish Talbot, Lady Mary, Countess of Shrewsbury

Talbot Herbert, Lady Catherine, Countess of Pembroke 298

Talbot Herbert, Lady Mary, Countess of Pembroke see Herbert, Lady Mary Talbot, Countess of Pembroke

Tate, Nahum 114

Tatius, Achilles see Achilles Tatius theatre (theatrical) 58, 100, 113, 122, 132-3, 150-1, 215, 218, 221, 236, 240-1, 253, 258, 283, 290-3, 310

comedy 154, 202, 209, 211, 238 drama 2, 6, 12, 16, 20, 33, 35, 38, $43,60-1,65,83,85,113,126$,
$132-5,138,146,150-1,156-7$, $161,169,183,185,215,218-19$, 231-2, 235, 238-9, 245-6, 253-4, 264, 273, 303 masque $6,20,35,41,43,149,170$, 195-6, 216, 218, 265, 275, 277, 279-80, 284, 308-13, 328, 334, 337

play $6,126,144,161,169,218$, 225-6, 232, 235, 238, 245, 248-9, 252-4, 256, 258, 260, 278, 297, 337

scene $38,46-7,54,56,58,77-8$, 86-7, 100-1, 103, 106-7, 125, 130-2, 135, 142, 144-6, 149, $150,157,160,181,183,185$, 187, 195-7, 200, 205-8, 219, $222,225,245,258,265,282$, 284-5, 297, 334

stage $86,109,113,132-3,150-1$, 216, 219-20, 224, 235, 245, 275, 277-8, 283, 300-1

tragedy $133-4,151,184,186,211$, 218-19, 238, 245-6, 253, 264, 306

The Merchant of Venice see Shakespeare

Theodosius 164

Theophrastus (Theophrastan) 4, 15-18, 49, 56, 106, 129, 152, $181,185-6,211,224,258,332$

Theseus 240

The Winter's Tale see Shakespeare Thisbe 113, 127, 135-6, 141, 145-6, 200, 210

Thornley, George 5, 34, 36-9, 44, 48-9, 51-2, 54-5, 57-60, 63, 73,338

Thucydides 258-9

Tirol, Ferdinand of 124, 192

Toutain, Jacques 33

Tower Hill 45, 305

tragedy see theatre

Trajan 233 
Treaty of Blois 30, 228

Treaty of London 253

Trent, Council of (tridentine) see Council of Trent

Trentham de Vere, Lady Elizabeth, Countess of Oxford 294

Troy, Helen of 186

Tudor, Elizabeth I 2, 25, 35, 41-5, 61, $74,109,123,170-1,191-2,212$, 222, 228, 237, 242-51, 267, 272, 291, 324

Tudor, Henry VIII 298

Tudor, Mary I 175, 255-6

Turenne, Viscount of see La Tour d'Auvergne, Henri de, Viscount of Turenne

Turenne, third Viscountess of see Orange-Nassau-Dillenburg de La Tour d'Auvergne, Elisabeth (Elizabeth), Princess of Nassau, third Viscountess of Turenne

Turnhout, Battle of 245

Tuscon 171

Tyranny (tyrannise, tyrannize, tyrannous) $1-3,13,110,115,117$, $125,129,136,139,148,153$, 161-2, 174-80, 187, 189-90, $212,214,224-5,232,235$, 237-8, 248-50, 256-65, 267, 270-2, 285, 313-18, 321, 324, 339

Tyras 171

Tyron, Antoine 337

Underdowne (Vnderdoune, Vnderdowne), Thomas 5, 120-2, $126-7,129,132-4,136-44$, $147-8,151-62,164-5,179$

Urania see Sidney Wroth Ursinus, Fulvius see Orsini, Fulvio Ursinus, Zacharias 116 Utrecht, Union of 177

Valois Bourbon, Marguerite de,
Queen of France 30, 239, 336

Valois, Charles IX 29-30, 69, 117, 227

Valois, François I 24, 28-9

Valois, François II 29-30

Valois, François Hercule, Duc d'Anjou 117

Valois, Henri (Henry) de 30, 32, 228, 237-9, 241-2, 244-5, 271

van de Passe, Simon 277

Vascosan, Michel de 142, 226-7, 229

Vautrollier, Thomas 227

Venus 76, 99, 105, 141, 153, 173, 184, 200, 223, 227, 302, 329, 331

Vere, Lady Anne Cecil de, Countess of Oxford see Cecil de Vere, Lady Anne, Countess of Oxford

Vere, Edward de, seventeenth Earl of Oxford 35, 121, 245, 282, 294

Vere, Lady Elizabeth Trentham de, Countess of Oxford see Trentham de Vere, Lady Elizabeth, Countess of Oxford

Vere, Sir Francis 245

Vere, Henry de, eighteenth Earl of Oxford 294

Vere, Sir Horace 245

Vere Herbert, Lady Susan de, Countess of Pembroke and Montgomery 252, 282, 290, 292, 294-5, 304, 329

Veronese 177

Vienne, P. de 66

Vigenère (Vigenere), Blaise de 14

Villiers, George, Duke of Buckingham 178,303

Vindiciae, contra tyrannos 175-6, 267,318

Virgil 33, 186, 301

virgin (virgine, virginity, virginitie) see erotica

Viterbo, Annius of 164, 168, 171-2

Volumnius 233

Wales (Welsh) 331 
Walsingham, Sir Francis 42-3, 46, 179, 224, 226

Walsingham Sidney Devereux Burke, Lady Frances, Countess of Clanricard and St. Albans 44-6, 204, 301, 318

Wars of Religion 1, 238, 246, 248, 271

Warschewiczki, Stanislaus 5, 20, 115-18, 120-1, 123, 129, 132-4, 136-44, 147-8, 151-2, $154-8,161,165,174$

Wechel, Johann 71-2, 116

White Mountain, Battle of the 123-4

Whitehall 42-3

Whyte, Rowland 245

William I 'The Silent', Prince of

Orange see Orange-NassauDillenburg, Willem I, Prince of Wilson, Arthur 274

Wotton, Edward, first Baron Wotton 251

Wotton, Sir Henry 249, 251

Wright, James 52

Wriothesley, Henry, third Earl of Southampton $25,35,70,80$, 98,251
Wroth, James 274

Wroth, Katherine 287

Wroth, Lady Margaret Rich see Rich Wroth, Lady Margaret

Wroth, Lady Mary see Sidney Wroth, Lady Mary

Wroth, Lady Mary Rich see Sidney Wroth, Lady Mary

Wroth, Sir Robert (c. 1539-27 January 1606) 300

Wroth, Sir Robert (c. 1576-1614) 281, 290-1, 300-1

Wroth, Lady Susan Stonard see Stonard Wroth, Lady Susan

Wroth, Sir Thomas (1518?-73) 300

Wroth, Sir Thomas (1584-1672)

$$
\text { 301-2, } 309
$$

Wroth, William 287

Wyatt's Rebellion 175

Wycherley, William 49-50

Xenophon of Ephesus 35

York House 242

zeugma see rhetoric 